\title{
Constant Weight Codes with Package CodingTheory.m in Mathematica
}

\author{
Igor Gashkov \\ Karlstad University, Department of Engineering Sciences, Physics and Mathematics 65188 \\ Karlstad Sweden \\ Igor.Gachkov@kau.se
}

\begin{abstract}
The author offers the further development of package CodingTheory.m [1] in the direction of research of properties and parameters of Constant weight codes (lower and upper bounds) based on works [2], [3] and also using the Table of Constant Weight Binary Codes (online version, Neil J. A. Sloane: Home Page http://www.research.att.com/ njas/codes/Andw/) and the table of upper bounds on $A(n, d, w)$ (which in many cases also gives lower bounds) maintained by Erik Agrell, Alexander Vardy and Kenneth Zeger, and which is an electronic supplement to their paper Upper bounds for constantweight codes, http://www.s2.chalmers.se/ agrell/bounds/cw.html. The offered package allows to carry out the comparative analysis of parameters of new codes with classical upper bounds such as Johnson bound, Linear programming (Ph Delsarte) bound ..., and also with already available classes of codes. As an example we consider a some construction of codes as union of two codes with parameters $(n, 2 a, a+b)$ and $(m, 2 b, a+b)$, that in some cases gives codes the best than codes obtained with use Juxtaposing .
\end{abstract}

\section{Introduction}

An $(n, d, w)$ constant weight binary code is a binary code of length $n$, code distance $d$ in which all code words have the same number, $w$, of "ones." We will denote the maximal possible size of $(n, d, w)$ constant weight code by $A(n, d, w)$. The most important and interesting problem is finding the largest possible size $A(n, d, w)$ of a $(n, d, w)$ constant weight code (hereafter called optimal codes). The results of code searching used to be put in tables of optimal codes. The first lower bound appeared in 1977 in the book of MacWilliams and Sloane ([4], pp.684-691). A table of binary constant weight codes of length $n \leq 28$ with explicit constructions for most of the 600 codes was presented in the encyclopedic work of Brouwer, Shearer, Sloane, and Smith [2]. Today Neil J. A. Sloane presents his table of constant weight codes online and performs continual updates. There is a companion table of upper bounds on $A(n, d, w)$ (which in many cases also gives lower bounds) maintained by Erik Agrell, Alexander Vardy and Kenneth Zeger, and which is an electronic supplement to their paper Upper bounds for constant-weight codes. The fragment of Sloane's table of constant weight codes with parameters $20 \leq n \leq 70, d=18$ and $10 \leq w \leq 16$ looks as 
follows (the point means that the appropriate code is optimal code and index give the key till example "j" means Juxtaposing (see below))

\begin{tabular}{|c|c|c|c|c|c|c|c|}
\hline \multicolumn{8}{|c|}{$\begin{array}{l}\text { TABLE } \mathrm{d}=18 \\
\text { Lower bounds on } \mathrm{A}(\mathrm{n}, 18, \mathrm{w})\end{array}$} \\
\hline $\mathbf{n}, \mathbf{w}$ & 10 & 11 & 12 & 13 & 14 & 15 & 16 \\
\hline 20 & 2. & 2 & 1 & 1 & 1 & $?$ & $?$ \\
\hline$\ldots$ & $\ldots$ & $\ldots$ & $\ldots$ & $\ldots$ & $\ldots$ & $\ldots$ & $\ldots$ \\
\hline 26 & 2. & 2. & 2. & 2. & 2 & $?$ & $?$ \\
\hline 27 & 3. ${ }^{j}$ & $3 .^{j}$ & $3 .^{j}$ & $3 .^{j}$ & 3 & $?$ & $?$ \\
\hline 28 & 3. & 3. & 3. & 4. ${ }^{\mathrm{j}}$ & $4 .^{j}$ & $?$ & $?$ \\
\hline$\ldots$ & $\ldots$ & $\ldots$ & $\ldots$ & $\ldots$ & $\ldots$ & $\ldots$ & $\ldots$ \\
\hline 69 & $18 .^{\mathrm{z} 18}$ & $?$ & $?$ & $?$ & $?$ & $?$ & $?$ \\
\hline 70 & $21 .^{\mathrm{z} 18}$ & $?$ & $?$ & $?$ & $?$ & $?$ & $?$ \\
\hline $\mathbf{n}, \mathbf{w}$ & 10 & 11 & 12 & 13 & 14 & 15 & 16 \\
\hline
\end{tabular}

\section{The Package "Constant Weight Codes"}

The package " Constant Weight Codes" is a file written in MATHEMATICA and will be read into MATHEMATICA with the commands.

In [1] := <<ConstantWeightCodes

The package consists of two parts: one part with Table of Lower bounds on $A(n, d, w)$ based on the Sloane's table with explanations

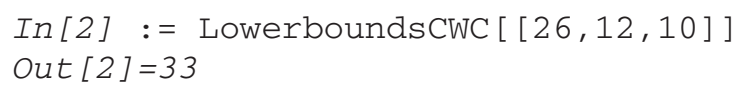

We can find information about this lower bound using command

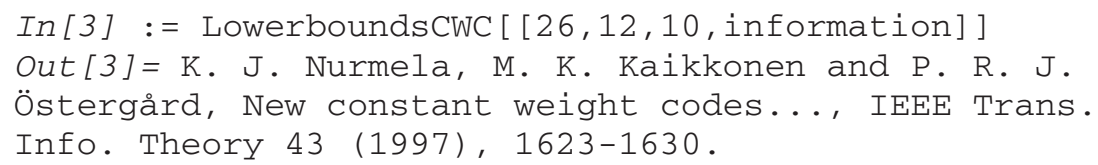

and the second part contains commands describing the classical upper bounds of constant weight codes. The information on the list of possible upper bounds can be received using the command ?*Bound*.

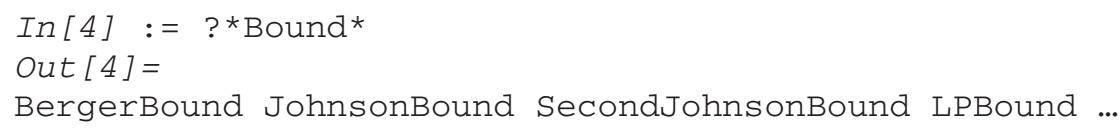

The complete information about a command is received by using the command ? Name. 
In $[5]:=$ ? LPBound

Out $[5]=$ LPBound $[\mathrm{n}, \mathrm{d}, \mathrm{w}]$ give Linear programming upper

bound ( $\mathrm{Ph}$. Delsarte) on the size for constant weight code $n$-length of code, d- code distance, d is even number and $w^{-}$weight of the code.

We can see also as far as the code (see $\operatorname{In}[2], \operatorname{In}[3]$, lower bound) differs from known upper bounds

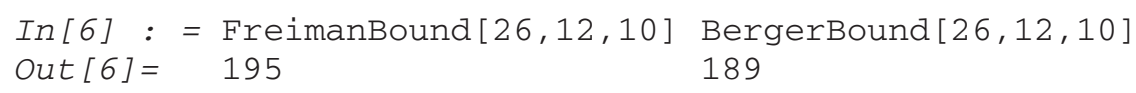

If the number of code vectors on a code coincides with one of the upper bound this give, that the code is optimal code

$$
\begin{array}{lll}
\text { In }[7]:= & \text { OptimalCWC }[[58,18,10]] & \text { OptimalCWC }[[70,18,10] \\
\text { Out }[7]= & ? & 21
\end{array}
$$

We also have an opportunity to make changes in a package, in case of occurrence of new codes with parameters it is better known

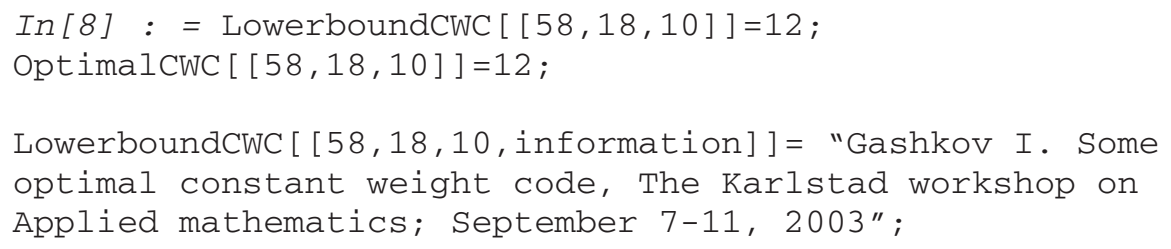

LowerboundCWC $[[58,18,10$, information $]]=$ "Gashkov I. Some optimal constant weight code, The Karlstad workshop on Applied mathematics; September 7-11, 2003";

DeleteFile [ "LowerboundCWC" , "OptimalCWC"] ; Save [Lowerboun dCWC", LowerboundCWC] ; Save [ "OptimalCWC" , OptimalCWC] ;

Now we will show how, using MATHEMATICA, we can, base on Sloane's table (see above) update this table. Trivial values we can obtain from well knows theorem 1. [2]

\section{Theorem 1.}

a) If $\mathrm{d}$ is odd, $A(n, d, w)=A(n, d+1, w)$.

b) $A(n, d, w)=A(n, d, n-w)$

c) $A(n, d, w)=1$ if $2 w<d$

d) If $d=2 w$ then $A(n, d, w)=[n / w]$, where $[n / w]$ is greatest integer less then or equal $n / w$.

e) $A(n, 2, w)=\left(\begin{array}{l}n \\ w\end{array}\right)$.

By juxtaposing two codes (the method of construction a new codes by placing them side by side) we can obtain lower bound $A(n 1+n 2, d 1+d 2, w 1+w 2) \geq \min \{A(n 1$, $d 1, w 1), A(n 2, d 2, w 2)\}$. Codes obtain with use juxtaposing we shall denote through $m$ $(n 1, d 1, w 1)+(n 2, d 2, w 2)$ where $m$ is $\min \{A(n 1, d 1, w 1), A(n 2, d 2, w 2)\}$. The command Juxtaposing[...] changes the table of codes supplementing with its codes obtained above by a stated method, in a case if the " $\mathrm{j}$ " code has the greater or equal 
number of code vectors, than already available in the table. As example we chose the parameters of the table $20 \leq n \leq 70, d=18$ and $10 \leq w \leq 16$.

In [9]:= LowerboundsCWC = Juxtaposing[LowerboundsCWC];

We chose the parameters of the table $20 \leq \mathrm{n} \leq 70, \mathrm{~d}=18$ and $10 \leq \mathrm{w} \leq 16$.

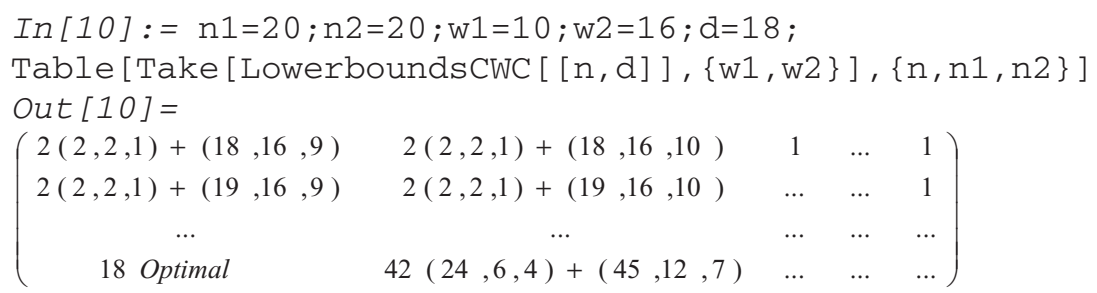

We can give the next generation of this table of lower bounds, using the following theorem.

Theorem 2. If $A(n 1,2 a, a+b)=M 1$ and $A(n 2,2 b, a+b)=M 2$ then $A(n 1+n 2,2(a+b), \min \{n 1, n 2\}) \geq \min \{M 1, M 2\}+1$

Proof. Let $n 1 \geq n 2$. We placing two codes $(n 1,2 a, a+b)$ and $(n 2,2 b, n 2-a-b)$ side-by-side. Using Juxtaposing we obtain code with parameters $(n 1+n 2,2(a+b), n 2)$. But we can add to code one binary vector more $v=(0,0, \ldots 0,1,1, \ldots 1)$, where the first $n 1$ coordinates are equal 0 , the last $n 2$ equal 1 . We can calculate distance between any vectors belonging to code $(n 1+n 2,2(a+b), n 2)$ and vector $v$. We can calculate this distance as the sum of two distances: the first distance is the distance between an any vector of a code $(n 1,2 a, a+b)$ and a zero vector, the second is the distance between an any vector of a code $(n 2,2 b, n 2-a-b)$ and a "one "vector (vector consisting of 1 ).

Example: Codes with parameters $(6,4,3)$ and $(4,2,3)$ consist of the same number of vectors. Code $(6,4,3) \quad$ Code $(4,2,3)$

$$
\begin{array}{llll}
\mathrm{V} 1=(1,1,1,0,0,0) & (1,1,1,0)=\mathrm{W} 1 & (1,1,1,0,0,0, & 0,0,0,1) \\
\mathrm{V} 2=(0,0,1,1,1,0) & (1,1,0,1)=\mathrm{W} 2 & (0,0,1,1,1,0, & 0,0,1,0) \\
\mathrm{V} 3=(0,1,0,1,0,1) & (0,1,1,1)=\mathrm{W} 3 & (0,1,0,1,0,1, & 1,0,0,0) \\
\mathrm{V} 4=(1,0,0,0,1,1) & (1,0,1,1)=\mathrm{W} 4 & (1,0,0,0,1,1, & 0,1,0,0) \\
& & (0,0,0,0,0,0, & 1,1,1,1)
\end{array}
$$

Code $(10,6,4)$

We shall denote such codes through $m(n 1,2 a, a+b) \cup(n 2,2 b, a+b)$ where $m$ is $\min \{M 1, M 2\}+1$. We can transform the table of constant weight codes taking into

account the theorem 2 and we obtain a new codes

$$
\begin{aligned}
& (13,6,8) \cup(20,10,8)->A(33,16,13) \geq 18 \\
& (13,6,8) \cup(21,10,8)->A(34,16,13) \geq 19 \\
& (16,8,9) \cup(19,10,9)->A(35,18,16) \geq 17 \\
& (13,6,9) \cup(23,12,9)->A(36,18,13) \geq 11
\end{aligned}
$$


$(13,6,9) \cup(24,12,9)->A(37,18,13) \geq 14$

$(8,2,6) \cup(31,10,6)->A(39,12,8) \geq 29$

$(14,6,9) \cup(25,12,9)->A(39,18,14) \geq 26$

$(14,6,9) \cup(26,12,9)->A(40,18,14) \geq 27$

$(14,6,9) \cup(27,12,9)->A(41,18,14) \geq 29$

$(11,2,8) \cup(57,14,8)->A(68,16,11) \geq 58$

Above the carried out computer calculations prove, that codes that we obtain using the theorem 2 it is impossible to present as juxtaposing two codes.

\begin{tabular}{|c|c|c|c|}
\hline $2(2,2,1)+(18,16,9)$ & $2(2,2,1)+(18,16,10)$ & 1 & 1 \\
\hline $2(2,2,1)+(19,16,9)$ & $2(2,2,1)+(19,16,10)$ & $2(2,2,1)+(19,16,11)$ & 1 \\
\hline $2(2,2,1)+(20,16,9)$ & $2(2,2,1)+(20,16,10)$ & $2(2,2,1)+(20,16,11)$ & $2(2,2,1)+(20,16,12)$ \\
\hline $2(2,2,1)+(21,16,9)$ & $2(2,2,1)+(21,16,10)$ & $2(2,2,1)+(21,16,11)$ & $2(2,2,1)+(21,16,12)$ \\
\hline $2(2,2,1)+(22,16,9)$ & $2(2,2,1)+(22,16,10)$ & $2(2,2,1)+(22,16,11)$ & $2(2,2,1)+(22,16,12)$ \\
\hline $2(2,2,1)+(23,16,9)$ & $2(2,2,1)+(23,16,10)$ & $2(2,2,1)+(23,16,11)$ & $2(2,2,1)+(23,16,12)$ \\
\hline $2(2,2,1)+(24,16,9)$ & $2(2,2,1)+(24,16,10)$ & $2(2,2,1)+(24,16,11)$ & $2(2,2,1)+(24,16,12)$ \\
\hline $3(3,2,1)+(24,16,9)$ & $3(3,2,1)+(24,16,10)$ & $3(3,2,1)+(24,16,11)$ & $3(3,2,1)+(24,16,12)$ \\
\hline $3(3,2,1)+(25,16,9)$ & $3(3,2,1)+(25,16,10)$ & $3(3,2,1)+(25,16,11)$ & $4(4,2,1)+(24,16,12)$ \\
\hline $3(3,2,1)+(26,16,9)$ & $3(3,2,1)+(26,16,10)$ & $3(3,2,1)+(26,16,11)$ & $4(4,2,1)+(25,16,12)$ \\
\hline $3(3,2,1)+(27,16,9)$ & $3(3,2,1)+(27,16,10)$ & $5(10,6,4)+(20,12,8)$ & $5(10,6,4)+(20,12,9)$ \\
\hline $3(3,2,1)+(28,16,9)$ & $3(3,2,1)+(28,16,10)$ & $5(10,6,4)+(21,12,8)$ & $5(4,2,2)+(27,16,11)$ \\
\hline $3(3,2,1)+(29,16,9)$ & $4(4,2,1)+(28,16,10)$ & $5(5,2,1)+(27,16,11)$ & $6(7,4,3)+(25,14,10)$ \\
\hline $3(3,2,1)+(30,16,9)$ & $4(4,2,1)+(29,16,10)$ & $6(7,4,3)+(26,14,9)$ & $7(0,4,3)+(26,14,10)$ \\
\hline $4(4,2,1)+(30,16,9)$ & $4(4,2,1)+(30,16,10)$ & $6(4,2,2)+(30,16,10)$ & $8(8,4,3)+(26,14,10)$ \\
\hline $4(4,2,1)+(31,16,9)$ & $5(5,2,1)+(30,16,10)$ & $7(7,4,3)+(28,14,9)$ & $10(15,8,6)+(20,10,7)$ \\
\hline $4(4,2,1)+(32,16,9)$ & $6(6,2,1)+(30,16,10)$ & $9(12,6,4)+(24,12,8)$ & $11 \mathrm{New}(13,6,9) \cup(23,12,9)$ \\
\hline $4(4,2,1)+(33,16,9)$ & $6(6,2,1)+(31,16,10)$ & $9(12,6,4)+(25,12,8)$ & $14 \operatorname{New}(13,6,9) \cup(24,12,9)$ \\
\hline $4(4,2,1)+(34,16,9)$ & $7(17,8,5)+(21,10,6)$ & $10(13,6,4)+(25,12,8)$ & $16(16,8,6)+(22,10,7)$ \\
\hline $4(4,2,1)+(35,16,9)$ & $7(17,8,5)+(22,10,6)$ & $13(13,6,4)+(26,12,8)$ & $16(16,8,6)+(23,10,7)$ \\
\hline $5(5,2,1)+(35,16,9)$ & $8(12,6,4)+(28,12,7)$ & $13(13,6,4)+(27,12,8)$ & $17(17,8,6)+(23,10,7)$ \\
\hline$S(5,2,1)+(36,16,9)$ & $8(13,6,4)+(28,12,7)$ & $14(14,6,4)+(27,12,8)$ & $20(16,6,4)+(25,12,9)$ \\
\hline $5(5,2,1)+(37,16,9)$ & $9(18,8,5)+(24,10,6)$ & $16(20,8,5)+(22,10,7)$ & $21(18,8,6)+(24,10,7)$ \\
\hline $5(5,2,1)+(38,16,9)$ & $9(12,6,4)+(31,12,7)$ & $16(20,8,5)+(23,10,7)$ & $24(19,8,6)+(24,10,7)$ \\
\hline $5(4,2,2)+(40,16,8)$ & $10(19,8,5)+(25,10,6)$ & $20(21,8,5)+(23,10,7)$ & $28(19,8,6)+(25,10,7)$ \\
\hline $6(6,2,1)+(39,16,9)$ & $12(19,8,5)+(26,10,6)$ & $21(21,8,5)+(24,10,7)$ & $28(19,8,6)+(26,10,7)$ \\
\hline $6(6,2,1)+(40,16,9)$ & $13(20,8,5)+(26,10,6)$ & $21(21,8,5)+(25,10,7)$ & $28(19,8,6)+(27,10,7)$ \\
\hline $6(6,2,1)+(41,16,9)$ & $14(20,8,5)+(27,10,6)$ & $23(23,8,5)+(24,10,7)$ & $36(20,8,6)+(27,10,7)$ \\
\hline $6(6,2,1)+(42,16,9)$ & $16(20,8,5)+(28,10,6)$ & $24(24,8,5)+(24,10,7)$ & $37(20,8,6)+(28,10,7)$ \\
\hline $7(0,2,1)+(42,16,9)$ & $16(20,8,5)+(29,10,6)$ & $25(19,8,6)+(30,10,6)$ & $37(21,8,6)+(28,10,7)$ \\
\hline $7(7,2,1)+(43,16,9)$ & $20(21,8,5)+(29,10,6)$ & $28(19,8,6)+(31,10,6)$ & $39(20,8,6)+(30,10,7)$ \\
\hline $7(0,2,1)+(44,16,9)$ & $21(21,8,5)+(30,10,6)$ & $31(20,8,6)+(31,10,6)$ & $40(20,8,6)+(31,10,7)$ \\
\hline $8(8,2,1)+(44,16,9)$ & $21(21,8,5)+(31,10,6)$ & $31(20,8,6)+(32,10,6)$ & $42(21,8,6)+(31,10,7)$ \\
\hline $8(8,2,1)+(45,16,9)$ & $23(23,8,5)+(30,10,6)$ & $31(21,8,6)+(32,10,6)$ & $42(22,8,6)+(31,10,7)$ \\
\hline $9(9,2,1)+(45,16,9)$ & $24(24,8,5)+(30,10,6)$ & $31(22,8,6)+(32,10,6)$ & $42(23,8,6)+(31,10,7)$ \\
\hline 11 Optimal z 17 & $25(25,8,5)+(30,10,6)$ & $35(20,8,6)+(35,10,6)$ & $42(24,8,6)+(31,10,7)$ \\
\hline$?$ & $30(25,8,5)+(31,10,6)$ & $35(21,8,6)+(35,10,6)$ & $42(25,8,6)+(31,10,7)$ \\
\hline$?$ & $30(25,8,5)+(32,10,6)$ & $35(22,8,6)+(35,10,6)$ & $42(26,8,6)+(31,10,7)$ \\
\hline$?$ & $31(27,8,5)+(31,10,6)$ & $35(23,8,6)+(35,10,6)$ & $42(27,8,6)+(31,10,7)$ \\
\hline$?$ & $31(27,8,5)+(32,10,6)$ & $38(15,6,5)+(44,12,7)$ & $42(14,6,6)+(45,12,7)$ \\
\hline$?$ & $31(28,8,5)+(32,10,6)$ & $42(15,6,5)+(45,12,7)$ & $45(15,6,6)+(45,12,7)$ \\
\hline ? & $31(29,8,5)+(32,10,6)$ & $45(16,6,5)+(45,12,7)$ & $45(16,6,6)+(45,12,7)$ \\
\hline$?$ & $31(27,8,5)+(35,10,6)$ & $45(17,6,5)+(45,12,7)$ & $45(17,6,6)+(45,12,7)$ \\
\hline$?$ & $33(28,8,5)+(35,10,6)$ & $45(18,6,5)+(45,12,7)$ & $48(15,6,6)+(48,12,7)$ \\
\hline$?$ & $34(29,8,5)+(35,10,6)$ & $48(16,6,5)+(48,12,7)$ & $56(15,6,6)+(49,12,7)$ \\
\hline ? & $35(30,8,5)+(35,10,6)$ & $48(16,6,5)+(49,12,7)$ & $56(16,6,6)+(49,12,7)$ \\
\hline$?$ & $37(22,6,4)+(44,12,7)$ & $56(17,6,5)+(49,12,7)$ & $56(17,6,6)+(49,12,7)$ \\
\hline $11(11,2,1)+(56,16,9)$ & $38(23,6,4)+(44,12,7)$ & $56(18,6,5)+(49,12,7)$ & $56(18,6,6)+(49,12,7)$ \\
\hline $12(12,2,1)+(56,16,9)$ & $40(23,6,4)+(45,12,7)$ & $56(19,6,5)+(49,12,7)$ & $57(11,4,5)+(57,14,8)$ \\
\hline 18 Optimal z 18 & $42(24,6,4)+(45,12,7)$ & $56(20,6,5)+(49,12,7)$ & $57(12,4,5)+(57,14,8)$ \\
\hline 21 Optimal z 18 & $45(25,6,4)+(45,12,7)$ & $57(13,4,4)+(57,14,8)$ & $60(22,8,6)+(48,10,7)$ \\
\hline
\end{tabular}

\begin{tabular}{|c|c|c|}
\hline 1 & 1 & 1 \\
\hline 1 & 1 & 1 \\
\hline 1 & 1 & 1 \\
\hline $2(2,2,1)+(21,16,13)$ & 1 & 1 \\
\hline $2(2,2,1)+(22,16,13)$ & $2(2,2,1)+(22,16,14)$ & 1 \\
\hline $2(2,2,1)+(23,16,13)$ & $2(2,2,1)+(23,16,14)$ & $2(2,2,1)+(23,16,15)$ \\
\hline $2(2,2,1)+(24,16,13)$ & $2(2,2,1)+(24,16,14)$ & $2(2,2,1)+(24,16,15)$ \\
\hline $3(3,2,1)+(24,16,13)$ & $3(3,2,1)+(24,16,14)$ & $3(3,2,1)+(24,16,15)$ \\
\hline $4(4,2,2)+(24,16,12)$ & $4(4,2,3)+(24,16,12)$ & $3(3,2,1)+(25,16,15)$ \\
\hline $4(4,2,1)+(25,16,13)$ & $4(4,2,2)+(25,16,13)$ & $4(4,2,3)+(25,16,13)$ \\
\hline $5(10,6,4)+(20,12,10)$ & $6(10,6,5)+(20,12,10)$ & $S(10,6,4)+(20,12,12)$ \\
\hline $6(7,4,3)+(24,14,11)$ & $6(4,2,2)+(27,16,13)$ & $6(4,2,2)+(27,16,14)$ \\
\hline $7(7,4,3)+(25,14,11)$ & $8(14,8,7)+(18,10,8)$ & $8(14,8,7)+(18,10,9)$ \\
\hline $9(15,8,6)+(18,10,8)$ & $11(11,6,5)+(22,12,10)$ & $11(11,6,5)+(22,12,11)$ \\
\hline $10(11,6,5)+(23,12,9)$ & $12(15,8,7)+(19,10,8)$ & $15(15,8,7)+(19,10,9)$ \\
\hline $12(16,8,6)+(19,10,8)$ & $16(16,8,6)+(19,10,9)$ & $17 \mathrm{New}(16,8,9) \cup(19,10$, \\
\hline $16(16,8,6)+(20,10,8)$ & $17(17,8,6)+(19,10,9)$ & $22(12,6,6)+(24,12,10)$ \\
\hline $17(17,8,6)+(20,10,8)$ & $22(12,6,6)+(25,12,9)$ & $24(13,6,6)+(24,12,10)$ \\
\hline $18(13,6,5)+(25,12,9)$ & $25(13,6,6)+(25,12,9)$ & $28(10,4,4)+(28,14,12)$ \\
\hline $26 \operatorname{New}(14,6,9) \cup(25,12,9)$ & $28(14,6,5)+(25,12,10)$ & $30(16,8,8)+(23,10,8)$ \\
\hline $27 \mathrm{New}(14,6,9) \cup(26,12,9)$ & $28(14,6,5)+(26,12,10)$ & $38(20,8,6)+(20,10,10)$ \\
\hline $29 \mathrm{New}(14,6,9) \cup(27,12,9)$ & $39(14,6,6)+(27,12,9)$ & $39(14,6,6)+(27,12,10)$ \\
\hline $39(15,6,5)+(27,12,9)$ & $39(14,6,6)+(28,12,9)$ & $45(19,8,7)+(23,10,9)$ \\
\hline $39(15,6,5)+(28,12,9)$ & $42(15,6,5)+(28,12,10)$ & $52(19,8,7)+(24,10,9)$ \\
\hline $39(16,6,5)+(28,12,9)$ & $48(16,6,5)+(28,12,10)$ & $56(20,8,7)+(24,10,9)$ \\
\hline $40(20,8,6)+(25,10,8)$ & $56(21,8,6)+(24,10,9)$ & $72(20,8,7)+(25,10,9)$ \\
\hline $48(21,8,6)+(25,10,8)$ & $56(21,8,6)+(25,10,9)$ & $80(20,8,7)+(26,10,9)$ \\
\hline $54(21,8,6)+(26,10,8)$ & $72(22,8,6)+(25,10,9)$ & $91(21,8,7)+(26,10,9)$ \\
\hline $56(21,8,6)+(27,10,8)$ & $78(20,8,7)+(28,10,8)$ & $118(21,8,7)+(27,10,9)$ \\
\hline $66(22,8,6)+(27,10,8)$ & $78(21,8,7)+(28,10,8)$ & $120(21,8,7)+(28,10,9)$ \\
\hline $77(22,8,6)+(28,10,8)$ & $78(22,8,7)+(28,10,8)$ & $132(22,8,7)+(28,10,9)$ \\
\hline$n(23,8,6)+(28,10,8)$ & $91(25,8,6)+(26,10,9)$ & $132(23,8,7)+(28,10,9)$ \\
\hline $78(24,8,6)+(28,10,8)$ & $100(25,8,6)+(27,10,9)$ & $132(24,8,7)+(28,10,9)$ \\
\hline $78(25,8,6)+(28,10,8)$ & $118(26,8,6)+(27,10,9)$ & $132(25,8,7)+(28,10,9)$ \\
\hline $78(26,8,6)+(28,10,8)$ & $130(26,8,6)+(28,10,9)$ & $167(22,8,7)+(32,10,9)$ \\
\hline $78(27,8,6)+(28,10,8)$ & $130(27,8,6)+(28,10,9)$ & $176(22,8,7)+(33,10,9)$ \\
\hline $78(28,8,6)+(28,10,8)$ & $130(28,8,6)+(28,10,9)$ & $198(23,8,7)+(33,10,9)$ \\
\hline$?$ & $?$ & $232(23,8,7)+(34,10,9)$ \\
\hline$?$ & $130(26,8,6)+(32,10,9)$ & $253(23,8,7)+(35,10,9)$ \\
\hline$?$ & $130(26,8,6)+(33,10,9)$ & $253(23,8,7)+(36,10,9)$ \\
\hline$?$ & $130(26,8,6)+(34,10,9)$ & $254(25,8,7)+(35,10,9)$ \\
\hline $78(13,2,2)+(48,16,12)$ & $130(26,8,6)+(35,10,9)$ & $257(26,8,7)+(35,10,9)$ \\
\hline $78(13,2,2)+(49,16,12)$ & $130(26,8,6)+(36,10,9)$ & $273(27,8,7)+(35,10,9)$ \\
\hline $78(13,2,2)+(50,16,12)$ & $130(11,2,3)+(52,16,12)$ & $278(27,8,7)+(36,10,9)$ \\
\hline $91(14,2,2)+(50,16,12)$ & $137(32,8,6)+(32,10,9)$ & $296(28,8,7)+(36,10,9)$ \\
\hline $100(15,2,2)+(50,16,12)$ & $137(32,8,6)+(33,10,9)$ & $300(29,8,7)+(36,10,9)$ \\
\hline $100(15,2,2)+(51,16,12)$ & $150(33,8,6)+(33,10,9)$ & $320(30,8,7)+(36,10,9)$ \\
\hline $105(15,2,2)+(52,16,12)$ & $150(33,8,6)+(34,10,9)$ & $327(30,8,7)+(37,10,9)$ \\
\hline $120(16,2,2)+(52,16,12)$ & $163(34,8,6)+(34,10,9)$ & $362(31,8,7)+(37,10,9)$ \\
\hline $130(17,2,2)+(52,16,12)$ & $163(34,8,6)+(35,10,9)$ & $372(32,8,7)+(37,10,9)$ \\
\hline $130(13,2,2)+(53,16,12)$ & $178(35,8,6)+(35,10,9)$ & $403(32,8,7)+(38,10,9)$ \\
\hline
\end{tabular}

In particular, codes that were obtain using the theorem 2 is close to optimal codes. For instance

$$
\begin{aligned}
& 28 \geq A(35,18,16) \geq 17 ; 12 \geq A(36,18,13) \geq 11 \\
& 15 \geq A(37,18,13) \geq 14 ; 39 \geq A(39,18,14) \geq 26
\end{aligned}
$$




\section{References}

1. I Gachkov "Error Correcting codes with Mathematica", Lecture note in Computer science LNCS 2657 s.737-746 (2003)

2. E. Brouwer, J.B. Shearer, N.J.A .Sloane, “A new table of Constant weight codes". IEEE Transactions of information theory, v 36, No 6 (1990)

3. E.Agrell,A. Vardy, and K. Zeger,"Upper bounds for constant-weight codes," IEEE Transactions on Information Theory,vol. 46, no. 7, pp. 2373-2395, Nov. 2000.

4. F.J. MacWilliams and N.J.A. Sloane, "The Theory of Error-Correcting Codes", Amsterdam: North-Holland, 1977 